\title{
Simulación de poblaciones celulares en dispositivos Organ-On- Chip: de los modelos clásicos a las simulaciones guiadas por datos.
}

\author{
Jacobo Ayensa-Jiménez*1, Marina Pérez-Aliacar ${ }^{1}$, Teodora Randelovic ${ }^{1}$, Sara Oliván ${ }^{1}$, \\ Iñaki Ochoa ${ }^{1}$, Mohamed Hamdy Doweidar ${ }^{1,2}$, Manuel Doblaré ${ }^{1,2}$

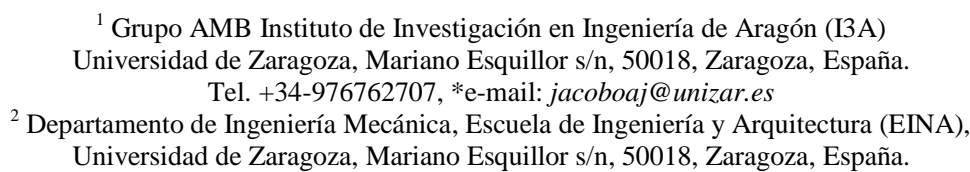

\section{Introducción}

Los microentornos celulares multi fenotípicos que se reproducen en dispositivos microfluídicos OrganOn-Chip son sistemas biológicos extremadamente complejos de modelar. Para diferentes poblaciones celulares, se deben considerar las interacciones químicas con nutrientes $\mathrm{u}$ otras señales, la disposición espacial, la rigidez y características del sustrato, la advección y difusión de fluidos y procesos celulares como la proliferación, la migración, la diferenciación y la muerte [1-2]. Todos estos fenómenos están altamente acoplados, lo que da lugar en un problema no lineal muy complejo de resolver.

Los modelos matemáticos son herramientas poderosas para comprender la complejidad de estos fenómenos [3-6]. Ofrecen una forma económica y flexible de explorar estrategias terapéuticas o la evaluación de fármacos en enfermedades como el cáncer. En este trabajo, se presenta un enfoque para modelar y simular el comportamiento de cultivos celulares en condiciones que puedan suponerse unidimensionales, capaz de capturar todos los procesos implicados. La metodología propuesta se ilustra en el estudio de microambientes de tumores celulares heterogéneos, en particular, el tumor cerebral más agresivo como es el glioblastoma, con objeto de comprender y cuantificar fenómenos tan importantes en su desarrollo como la formación de las estructuras celulares migratorias, conocidas como pseudoempalizadas o la formación de núcleos necróticos [7]. Los resultados obtenidos "in silico" están en línea con los resultados experimentales obtenidos en nuestros dispositivos microfluídicos de Organ-On-Chip donde se recrea el microambiente tumoral. Estas características metodológicas pueden extrapolarse al estudio de otros problemas de evolución tumoral en el que los factores epigenéticos, como los inducidos por la hipoxia, podrían desempeñar un papel importante.

\section{Materiales y Métodos}

Se presenta un enfoque matemático basado en las ecuaciones del continuo para el problema de la interacción de las diferentes poblaciones celulares y el oxígeno como especie química reguladora (aunque fácilmente generalizable a un número mayor de especies químicas involucradas). Las ecuaciones de gobierno son las ecuaciones de transporte (advección-difusión-reacción) para las poblaciones y las especies involucradas. Además, los términos de quimiotaxis, mecanotaxis, proliferación y diferenciación se consideran en las ecuaciones de balance. Cada uno de estos fenómenos puede, en general, expresarse como una función de las variables de campo (lo que hace que el problema sea no lineal). Más concretamente, se plantea la conservación de la masa de cada población y de oxígeno:

$$
\begin{array}{r}
\frac{\partial \boldsymbol{c}}{\partial t}=\frac{\partial}{\partial x}\left(\boldsymbol{f}\left(x, t, \boldsymbol{c}, \frac{\partial \boldsymbol{c}}{\partial x}, O_{2}, \frac{\partial O_{2}}{\partial x}\right)\right) \\
+\boldsymbol{s}\left(x, t, \boldsymbol{c}, \frac{\partial \boldsymbol{c}}{\partial x}, O_{2}, \frac{\partial O_{2}}{\partial x}\right) \\
\frac{\partial O_{2}}{\partial t}=\frac{\partial}{\partial x}\left(g\left(x, t, \boldsymbol{c}, \frac{\partial \boldsymbol{c}}{\partial x}, O_{2}, \frac{\partial O_{2}}{\partial x}\right)\right) \\
+r\left(x, t, \boldsymbol{c}, \frac{\partial \boldsymbol{c}}{\partial x}, O_{2}, \frac{\partial O_{2}}{\partial x}\right)
\end{array}
$$

donde $\boldsymbol{f}$ y $g$ son términos de flujo y $\boldsymbol{s}$ y $r$ términos fuente que deben ser definidos a partir de modelos paramétricos y ensayos experimentales.

El método se implementa utilizando el software MATLAB que ofrece la oportunidad al usuario de personalizar fácilmente este marco general para diferentes fenómenos.

\section{Resultados}

Las simulaciones son capaces de reproducir los resultados experimentales para un abanico importante de configuraciones en dispositivos microfluídicos (Figura 1). Fenómenos como la quimiotaxis inducida por hipoxia o la saturación celular demuestran tener un papel fundamental en la migración de las poblaciones (Figura 1a) y la formación de un core necrótico (Figura 1b) y pueden ser utilizados como casos clave para el diseño de 
terapias o pautas de actuación como respuesta a los fenómenos tumorales.

Ambos fenómenos (migración y necrosis) explican lo que en el campo de estudio del glioblastoma se conoce como pseudoempalizadas: movimientos celulares que se desplazan desde el tejido necrótico a las zonas más oxigenadas. Estas estructuras tan características pueden ser reproducidas in silico (Figura 2).

\section{Conclusiones}

La combinación de dispositivos de Organ-On-Chip y modelos computacionales resulta una herramienta muy útil para el planteamiento de nuevos modelos biológicos complejos que incluyen difusión, advección, quimiotaxis, mecanotaxis, proliferación, diferenciación y muerte celular.

No obstante, la enorme cantidad de parámetros que es necesario determinar para caracterizar los diferentes fenómenos, extremadamente acoplados y no lineales, hace natural el uso de herramientas del ámbito de la ciencia de datos y la inteligencia artificial para una mejor caracterización del problema [8]. Esta estrategia es la ideal en el marco de los estudios en dispositivos microfluídicos, donde la disponiblidad de una ingente cantidad de marcadores bioquímicos, imágenes y sensores, asegura un aporte importante de datos.

\section{AGRADECIMIENTOS}

Los autores agradecen el apoyo financiero del Ministerio de Economía y Competitividad de España (MINECO MAT201676039-C4-4-R, AEI / FEDER, UE).

\section{REFERENCIAS}

[1]. MOUSAVI, Seyed Jamaleddin; DOBLARÉ, Manuel; DOWEIDAR, Mohamed Hamdy. Computational modelling of multi-cell migration in a multi-signalling substrate. Physical biology, 2014, vol. 11, no 2, p. 026002.

[2]. MOUSAVI, Seyed Jamaleddin; DOWEIDAR, Mohamed Hamdy. Role of mechanical cues in cell differentiation and proliferation: a 3D numerical model. PloS one, 2015, vol. 10, no 5, p. e0124529.

[3]. MARTÍNEZ-GONZÁLEZ, Alicia, et al. Hypoxic cell waves around necrotic cores in glioblastoma: a biomathematical model and its therapeutic implications. Bulletin of mathematical biology, 2012, vol. 74, no 12, p. 2875-2896.

[4]. PAPADOGIORGAKI, Maria, et al. Mathematical modelling of spatio-temporal glioma evolution. Theoretical Biology and Medical Modelling, 2013, vol. 10 , no 1 , p. 47.

[5]. FRIEBOES, Hermann B., et al. Computer simulation of glioma growth and morphology. Neuroimage, 2007 , vol. 37, p. S59-S70.

[6]. CAI, Yan, et al. Mathematical modelling of a brain tumour initiation and early development: a coupled model of glioblastoma growth, pre-existing vessel cooption, angiogenesis and blood perfusion. PloS one, 2016, vol. 11, no 3, p. e0150296.

[7]. AYUSO, Jose M., et al. Glioblastoma on a microfluidic chip: Generating pseudopalisades and enhancing aggressiveness through blood vessel obstruction events. Neuro-oncology, 2017, vol. 19, no 4, p. 503-513.

[8]. AYENSA-JIMÉNEZ, Jacobo, et al. A new reliabilitybased data-driven approach for noisy experimental data with physical constraints. Computer Methods in Applied Mechanics and Engineering, 2018, vol. 328, p. $752-774$.

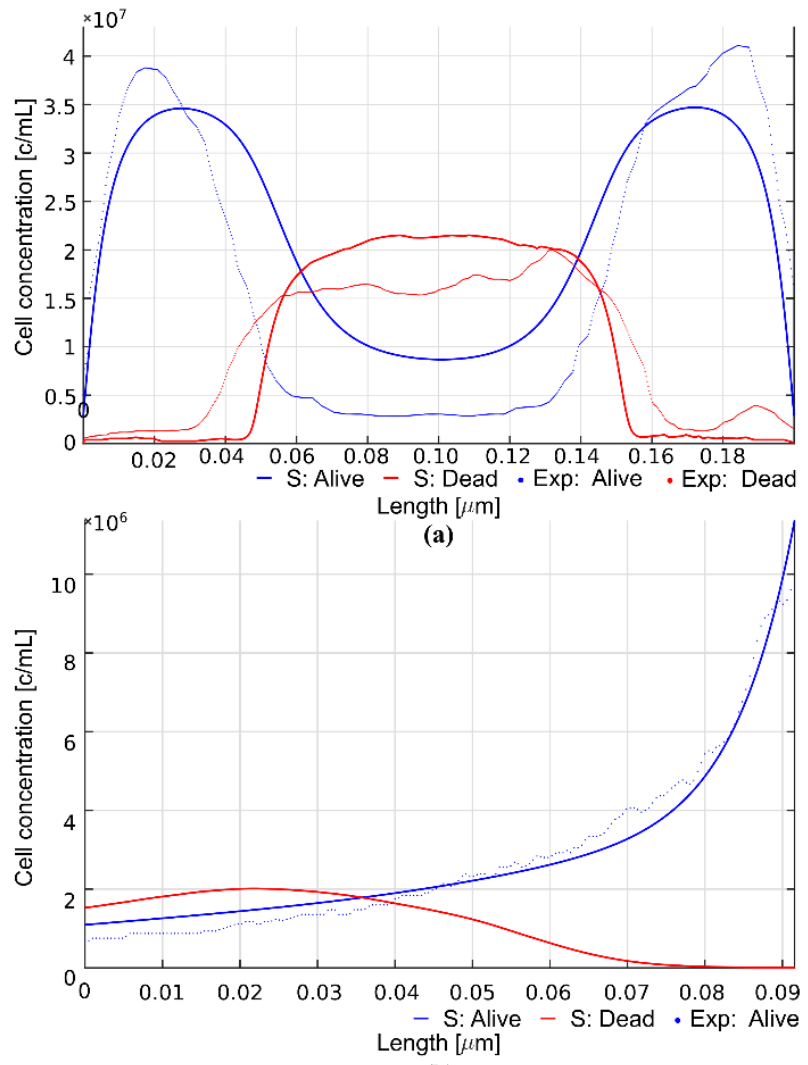

(b)

Fig 1. Reproducción de los experimentos. (a) Reproducción de la formación del core necrótico. (b) Reproducción de la migración inducida por la hipoxia

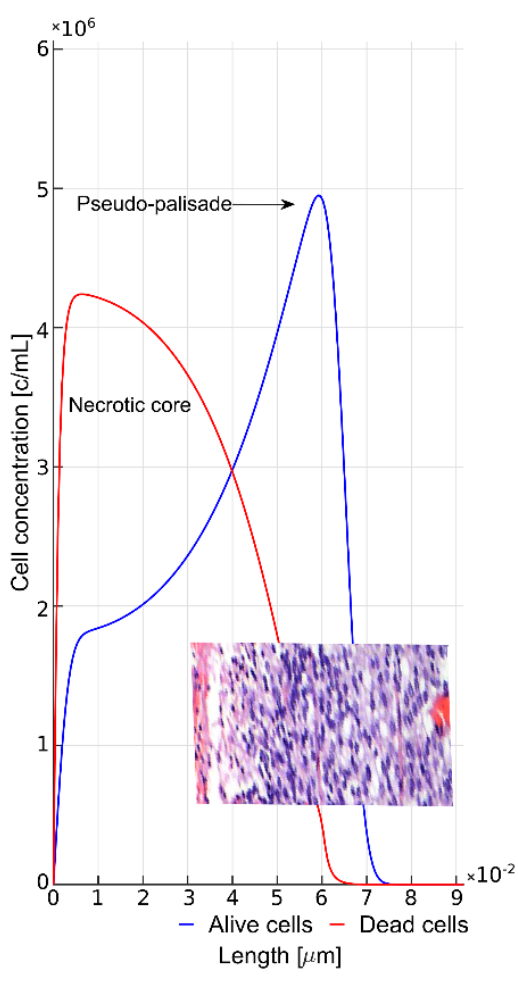

Fig 2. Recreación in silico de pseudoempalizadas 